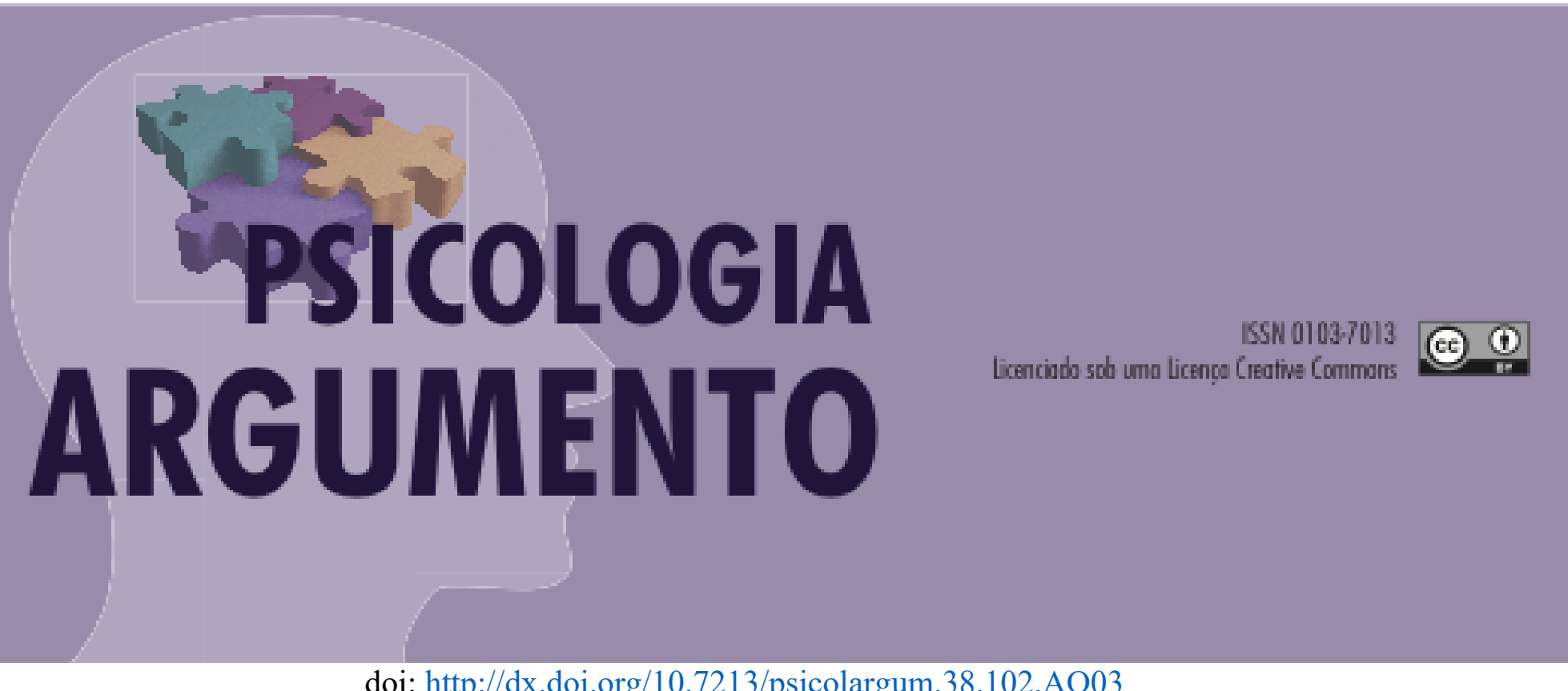

doi: http://dx.doi.org/10.7213/psicolargum.38.102.AO03

\title{
Bullying, preconceito e autoestima: discutindo as principais relações e distinções
}

Bullying, prejudice and self-esteem: discussing the main relationships and distinctions

Bullying, prejuicio y autoestima: discutir las principales relaciones y distinciones

Vitor José Araujo Matos

Mestrando em Psicologia pela Universidade Federal de Sergipe, vitor.psicologia10@hotmail.com, http://orcid.org/0000-0002-5283-0722

Joilson Pereira da Silva

Pós-doutor em Psicologia pela Universidade Autônoma de Barcelona, Docente e Pesquisador pela Universidade Federal de Sergipe: joilsonp@hotmail.com,

http://orcid.org/0000-0001-9149-3020

Karine David Andrade Santos

Mestre e Doutoranda em Psicologia pela Universidade Federal de Sergipe, psimulti@gmail.com,

http://orcid.org/0000-0001-9951-9539

Valéria Maria Azevedo Guimarães

Mestre e Doutoranda em Psicologia pela Universidade Federal de Sergipe, http://orcid.org/0000-0002-3782-4973

\section{Resumo}

O preconceito e o bullying são duas formas de violência escolar que podem atingir diferentes grupos socioculturais. São entendidos, na literatura científica, como fenômenos que causam prejuízos emocionais, cognitivos e comportamentais nos alvos da agressão, afetando a avaliação, 
ou autoestima, fator importante para o desenvolvimento individual e as relações interpessoais. Neste estudo, foi realizada uma revisão narrativa não-sistemática que objetivou apresentar e discutir os principais conceitos, relações e distinções entre preconceito, bullying e autoestima. Foi realizado um levantamento no banco de dados da Scielo, Pepsic e periódicos CAPES, através da plataforma $\mathrm{CAFe}$, de artigos científicos, dissertações, teses e livros, que promoviam o debate dos construtos apresentados, por meio dos descritores em português. Os resultados demonstraram que o preconceito e o bullying são fenômenos articulados que afetam, negativamente, a convivência, representando um perigo às escolas, devido as práticas agressivas serem percebidas como "brincadeiras", ocasionando a naturalização entre os grupos. Além disso, notou-se que a autoestima é uma variável importante no enfrentamento dessas condutas, por ser um indicativo para a criação de habilidades sociais que favorecem a saúde mental dos envolvidos.

Palavras-chave: escola, preconceito, bullying, autoestima, estudantes.

\begin{abstract}
Prejudice and bullying are two forms of school violence that can affect different socio-cultural groups. They are understood, in the scientific literature, as phenomena that cause emotional, cognitive, and behavioral damages in the targets of aggression, affecting the evaluation, or selfesteem, an important factor for individual development and interpersonal relationships. In this study, a non-systematic narrative review was carried out that aimed to present and discuss the main concepts, relationships, and distinctions between prejudice, bullying and self-esteem. A survey was carried out in the database of Scielo, Pepsic and CAPES journals, through the CAFe platform, of scientific articles, dissertations, theses, and books, which promoted the debate of the constructs presented, through the descriptors in Portuguese. The results showed that prejudice and bullying are articulated phenomena that negatively affect coexistence, representing a danger to schools, due to the aggressive practices being perceived as "games", causing naturalization between groups. In addition, it was noted that self-esteem is an important variable in coping with these behaviors, as it is indicative for the creation of social skills that favor the mental health of those involved.
\end{abstract}

Keywords: school, prejudice, bullying, self-esteem, students.

\title{
Resumen
}

Los prejuicios y el bullying son dos formas de violencia escolar que pueden afectar a diferentes grupos socioculturales. Se entienden, en la literatura científica, como fenómenos que causan daños emocionales, cognitivos y conductuales en los objetivos de agresión, que afectan la evaluación o la autoestima, un factor importante para el desarrollo individual y las relaciones interpersonales. En este estudio, se realizó una revisión narrativa no sistemática que tuvo como objetivo presentar y discutir los principales conceptos, relaciones y distinciones entre prejuicios, bullying y autoestima. Se realizó una encuesta en la base de datos de las revistas Scielo, Pepsic y CAPES, a través de la plataforma CAFe, de artículos cientificos, disertaciones, tesis y libros, que promovió el debate de los constructos presentados, a través de los descriptores en portugués. Los resultados mostraron que los prejuicios y el bullying son fenómenos articulados que afectan negativamente la convivencia, representando un peligro para las escuelas, debido a las prácticas agresivas que se perciben como "juegos", causando la naturalización entre los grupos. Además, se observó que la autoestima es una variable importante para hacer frente a estos comportamientos, ya que es indicativa de la creación de habilidades sociales que favorecen la salud mental de los involucrados

Palabras clave: escuela, prejuicio, bullying, autoestima, estudiantes.

\section{Introdução}


O bullying é um problema de saúde pública com uma crescente incidência nas escolas brasileiras. Conforme apontado pelo Instituto Nacional de Estudos e Pesquisas Educacionais Anísio Teixeira (INEP), em 2019, o Brasil é o país mais propício as condutas agressivas (10\%), estando acima da média internacional (3\%). O Programa Internacional de Avaliação de Estudantes (PISA), em 2017, estimou que um, em cada dez estudantes, sofre com as mais diversas categorias de bullying, sendo que 7,4\% são alvos de agressões físicas e verbais (zombaria, intimidação), na maior parte do tempo (Instituto Brasileiro de Geografia e Estatística, 2015).

O bullying é considerado um comportamento agressivo proativo caracterizado pela discrepância de poder entre os pares (Olweus, 2013), pautadas pela repetição, submissão e intenção de causar dano a um ou mais indivíduos (Zequinão, Medeiros, Lise, Trevisol, \& Pereira, 2019). Esta conduta pode ser movida pelo preconceito, ou seja, por ideias generalizadas e pré-julgamentos sociais, culturais e econômicas com os alvos delimitados, em uma combinação de aspectos cognitivos, emocionais e comportamentais (Crochík, 2019; Souza, 2013).

O preconceito e o bullying são fenômenos, apresentados por Antunes e Zuin (2008), como relacionados, sendo o preconceito a base do bullying. Crochík (2015), por sua vez, entende que são distintos, pois, no preconceito, há um alvo fixo, enquanto no bullying, não há essa especificidade, ou seja, qualquer um pode sofrer a agressão. Apesar das divergências teóricas, esses atos agressivos podem desencadear consequências de ordem psicológica, física e emocional que diminuem a autoestima dos estudantes (Bandeira \& Hutz, 2010; Chochík, 2019; Manzini \& Branco, 2017).

Levando em consideração que a autoestima é importante por ser um indicador de saúde mental (Schultheisz \& Aprile, 2013) e diante da relação entre bullying e preconceito, considerou-se pertinente realizar um levantamento bibliográfico da produção científica, na modalidade de revisão narrativa não-sistemática (Rother, 2007), acerca de bullying, preconceito e autoestima para responder o seguinte questionamento: Como essas três variáveis estão sendo investigadas? Levantou-se a hipótese que o preconceito é um fenômeno que se relaciona ao bullying, porém possui formas distintas e que ambos interferem, de forma negativa, a autoestima do estudante.

A coleta dos achados teóricos apresentados neste estudo foi conduzida em livros, teses, dissertações e artigos armazenados nas bases de dados da Scielo, Pepsic e CAPES, através da plataforma CAFe, com os descritores, em português, "bullying”, "preconceito" 
e "autoestima". O objetivo do estudo foi apresentar e discutir os principais conceitos, as relações e distinções entre preconceito, bullying e a autoestima.

\section{Relações e distinções entre preconceito, bullying e autoestima}

O preconceito e o bullying são formas de agressões mais praticadas dentro das escolas entre os estudantes (Crochík \& Crochík, 2017). Para definir o preconceito, diferentes modelos teóricos foram elaborados com esta finalidade. Crochík (2016) baseado na teoria crítica de Horkheimer e Adorno (1985), analisa-o como uma ideologia que se encontram a gênese dos estereótipos, das falsas generalizações, das valorizações que mobilizam o indivíduo a reproduzir na sociedade, características singulares que favorecem o desenvolvimento de atitudes hostis, aos que são tidos como mais frágeis, ocorrendo a agressão.

Nas escolas, Crochík e Crochík (2017) compreendem que essa relação é mediada pela hierarquia social que classifica e ordena, conforme as classes sociais e competências como: os alunos mais fortes e/ou mais fracos, os mais populares e os menos populares. Essa ideologia tem uma interação com a personalidade autoritária, uma vez que, no preconceito, quanto mais autoritário, maior a tendência do estudante praticar atos violentos em minorias e/ou pessoas em situação de inclusão, diminuindo as possibilidades de analisar e determinar suas próprias decisões, colocando em risco a si mesmo e aos outros.

Verificou-se que o indivíduo evita a identificação com seu alvo da agressão e nega qualquer desejo, medo ou ideais de forma semelhante (Crochík, 2015). Além disso, esse autor ressalta que essa percepção, que julga incorretamente as ações agressivas, fortalece o comportamento discriminatório, no qual pode se agravar devido a ser percebido como "brincadeiras". O comportamento discriminatório é uma resposta ao preconceito que cria, mantém ou reforça a existência das hierarquias, em alguns membros e/ou grupo, de maneira sutil ou não, como uma condição de vantagem sobre o outro com hostilidade, desprezo e agressão (Dovidio, Hewstone, Glick, \& Esses, 2010; Parker, 2012).

O preconceito é um pré-julgamento formado por uma combinação de componentes cognitivos, afetivos e disposicionais ou volitivos (Krech, Crutchfield, Ballachey, 1975; Lima, 2011; Crochík, 2019). Nos componentes cognitivos, estão contidos as crenças e os estereótipos, que estabelecem ideias, conhecimentos e expectativas generalizadas de um determinado grupo, atribuindo ao indivíduo características semelhantes, apenas por fazer 
parte dele. Essas percepções provocam reações afetivas que modificam os valores, as emoções e o comportamento do sujeito (Bodenhausen \& Richeson, 2010). Nas características disposicionais ou volitivas, manifestam-se pela segregação (exclui alguém ou um grupo da maioria ou de outros) e/ou marginalização (coloca-o à margem, ou seja, retira-o parcialmente do grupo). Crochík (2015) complementa que esses atributos têm uma tendência para a ação contra o alvo da agressão, sendo expressa pela hostilidade, pela superproteção e pela indiferença.

Esses elementos estão contidos em todos os tipos de preconceito, como: o racismo, a homofobia, o sexismo e, apesar das suas especificidades, apresentam em comum: 1) a ênfase nas relações de poder entre os grupos; 2) os sentimentos de antipatia contra alguém por não pertencer ao grupo; 3) ser de igual valor aos membros do grupo, alvos de preconceito; e 4) uma resistência social e cognitiva à desconfirmação das crenças e expectativas negativas em relação ao grupo (Lima, 2011). No estudo desenvolvido por Pettigrew e Meertens (1995), foi construída uma escala com dados de sete amostras independentes da Europa Ocidental, em quatro idiomas, para medir dois tipos de preconceitos: o flagrante e o sutil.

O preconceito flagrante se baseia na percepção de que outros grupos são uma ameaça de ordem emocional e econômica; por isso, devem ser rejeitados. No preconceito sutil, o indivíduo defende os valores tradicionais, excluindo aqueles que não possuem as condutas "adequadas", negam a cultura individual e qualquer emoção positiva, em relação aos outros. Estes dois tipos de preconceitos serviram para o desenvolvimento de pesquisas relacionadas ao racismo contra negros (Nunes, 2010), imigrantes (Herrera Rubalcaba \& Sánchez Quintero, 2013) e com profissionais do sexo (Imhoff, Dreizik, \& Brussino, 2020).

Em outro modelo para compreender o preconceito, Crandall e Esheman (2003), propõe a Justificação-Supressão, cujas características singulares de cada indivíduo levam à expressão e à experiência do próprio. São analisadas, nesse procedimento, a relação entre preconceito genuíno (não controlado e intrínseco) e preconceito explícito (controlado e flagrante). Nessa associação, foram indicados que alguns membros do grupo têm preconceito genuíno com força motivacional e, concomitantemente, afeta o autoconceito, aos valores e às crenças não preconceituosas, que suprimem uma expressão do preconceito. Além disso, essas expressões tendem a buscar uma imagem não preconceituosa, justificando as ações agressivas sem que sejam publicadas ou censuradas. 
O modelo da Discriminação Justificada, desenvolvido por Pereira, Vala e CostaLopes (2010), segue essa lógica da naturalização da agressão, uma vez que os indivíduos nutrem, em si, atos discriminatórios, porém se percebem como não sendo preconceituosos e tendem a justificar suas atitudes. Dessa forma, seus atos são percebidos como válidos e justos e, quanto mais os praticam, maior a legitimização em discriminar os grupos minoritários. Além disso, os indivíduos podem se intitular como inclusivos, ainda que tenham aversão à diferença do outro, pois, mesmo que não atribuam uma avaliação negativa e nem positiva, haverá, em seu grupo, o reconhecimento pelas ações prestadas (Souza, 2013).

Assim, essas contribuições teóricas apontam que o preconceito está articulado com os ambientes sociais, como a escola e a família, por envolvem a depreciação do outro, de maneira que, ocorra a desconfiguração da ordem social, na qual se fortalecem a cristalização no modo de pensar, de sentir e a falta de autonomia dos estudantes (Martins \& Kämpf, 2014). O estudo realizado por Cordeiro e Buendgens (2012) investigou os significados e os sentidos atribuídos às situações de preconceito nas escolas. Nos resultados, identificou-se que os estudantes apresentaram dificuldades em reconhecer os próprios sentimentos ao vivenciar o preconceito do que abordar, de maneira geral, a temática e, as ações preconceituosas provocavam o afastamento ou aproximação dos grupos, caracterizando a discriminação.

A discriminação na escola é uma prática individual e grupal, com ações e omissões que prejudicam a aprendizagem e influenciam, negativamente, a construção da identidade do estudante que podem ser levadas pelo decorrer da vida (Abramovay, 2015). O estudo realizado por Zunino, Bastos, Coelho, Mendes e Massignam (2015), com 1.023 graduandos da Universidade de Santa Catarina, analisou os aspectos relativos à discriminação (rótulos pejorativos, exclusão, desigualdade, considerar o outro como incapaz), conforme os dados sociodemográficos (idade, raça, posição socioeconômica, curso e gênero). Foram ressaltados que os estudantes de História, Pedagogia e Psicologia são os que mais sofrem com as atitudes discriminatórias, associados por serem pessoas mais velhas, mulheres, de pior posição socioeconômica, diferentes vestimentas, serem pretos e pardos.

As atitudes discriminatórias são realizadas intencionalmente quando o estudante é tratado de forma desigual em função de uma característica individual ou de grupo, que lhe é peculiar, tal como sua condição física (Crochík, 2015). Dessa forma, o bullying 
adentra ao preconceito e à discriminação, por estar associado ao processamento cognitivo e afetivo que, se considerado em uma cultura ampla, pode ocorrer a sua reprodução, tornando-se um desafio para as escolas por não somente de transmitir o conteúdo, mas também a convivência pacífica entre os alunos e o respeito às diferenças (Souza, 2013).

Alguns autores apresentam argumentos que relacionam o preconceito e o bullying, entre eles, Antunes (2010), Antunes e Zuin (2008) e Chaves e Souza (2018). Eles apontam que a falta de motivo aparente, a insistência e a intenção de causar dano ao indivíduo são pontos semelhantes entre esses fenômenos, defendendo que o preconceito é a base do bullying e envolve os fatores sociais que indicam os alvos da violência e, sobre os indicativos da função psíquica para aqueles que são os autores da agressão. Dessa forma, ressalta-se que o preconceito e o bullying são conceitos ligados por envolver os estereótipos e imagens preconcebidas (Martins \& Kämpf, 2014).

No entanto, Crochík (2015) sinaliza que esses fenômenos não podem ser entendidos como sinônimos, já que o alvo do preconceito é fixo e remete a um determinado grupo, com motivos justificados, especificados ou não, correspondentes a traços que são destinados à discriminação por parte da sociedade, a qual é diferenciada pela marginalização e segregação. Uma distinção entre os fenômenos está no tipo de alvo escolhido e a estrutura psíquica que envolve o autor da violência. No preconceito, o autor da agressão nega qualquer identificação com o seu alvo, seja pelas suas características e/ou sentimentos, com um estereótipo específico e, no bullying, qualquer sujeito pode ser o alvo, apenas pela sensação de dominar o outro (Crochík, 2016). Além disso, o bullying está mais relacionado com os aspectos comportamentais e nas relações interpessoais. No preconceito, ocorre nas relações intrapessoais e envolvem várias fases do desenvolvimento, como questões de gênero, origem étnica, idade e condição socioeconômica, atingindo os aspectos cognitivos, afetivos e comportamentais, não se limitando aos episódios de bullying (Souza, Silva, \& Faro, 2015).

As primeiras investigações do bullying tiveram destaque, a partir dos anos 90, com os estudos de Olweus (1993), Smith e Sharp (1994) e Rigby (1999). No Brasil, surgiram nos anos 2000 (Fante, 2005; Lisboa, 2005; Lopes Neto \& Saavedra, 2004) e servem de base, até hoje, para outras pesquisas que envolvem a prevalência de bullying em estudantes de escolas brasileiras (Malta, Mello, Prado, Sá, Marinho, Pinto, Silva, \& Silva, 2019; Oliboni, Lunardi, Lunardi, Pereira, \& Oliveira, 2019). Muito embora, o bullying não seja um fenômeno novo, sendo o resultado de uma sociedade que reforça o 
individualismo e a desvalorização da ética e respeito ao próximo, sofrendo influências de aspectos biológicos e culturais (Binsfeld \& Lisboa, 2010).

De acordo com a Lei $\mathrm{n}^{\circ} 3.185$, em vigor desde 2016, o bullying é uma intimidação sistemática, quando há violência física ou psicológica em atos de humilhação ou discriminação, incluindo ameaças, apelidos ofensivos, ataques físicos, entre outros (Brasil, 2015). Ele é um fenômeno multifacetado expresso de forma direta ou indireta e podem ocorrer em qualquer lugar, como escolas, asilos, penitenciárias, empresas, serviço público, clubes, entre outros (Manzini \& Branco, 2017; Rocha, Paixão, Teixeira, Paixão, Soares, \& Rocha, 2019; Teixeira, 2011). O bullying direto é o mais comum entre os meninos, por meio de agressões físicas e verbais. E, o bullying indireto, comum entre mulheres e crianças, é caracterizado pelo isolamento social e pela disseminação de rumores desagradáveis e desqualificantes. São tipificados como este tipo de conduta: 1) físico e material: bater, empurrões, puxar o cabelo, mandar outro colega agredir, estragar o material ou a roupa, pegar o material ou o dinheiro sem consentimento; 2) verbal: apelidos, xingamentos, ameaças, espalhar boatos, difamações; 3) social ou relacional: evitar ou ameaçar dizendo que não é mais seu colega se não fizer o que é mandado, excluir/rejeitar um colega do grupo; 4) sexual: exibicionismo, comportamentos de assédio, obscenos, insultar um colega devido à sua orientação sexual, divulgar comentários de caráter sexual; e 5) virtual ou cyberbullying: agredir, ameaçar, perseguir por meio de mídias sociais, caracterizado pelo anonimato (Martins \& Kämpf, 2014).

O bullying ocorre nas relações interpessoais e podem envolvem diferentes papéis sociais em relação ao comportamento praticado (Salmivalli, Lagerspetz, Björkqvist, Österman, \& Kaukiainen, 1996). Dentre esses papéis, são identificados o autor, alvo, alvo-autor e testemunha. Os autores da agressão possuem características de manipular o outro, geralmente, podem ser os mais populares, fortes e possuem um histórico sociofamiliar de violência, desafeto e reproduzem a condição preconceituosa experimentada (Fante, 2011). Enquanto os alvos podem apresentar características relacionadas a timidez, falta frequentemente às aulas, têm dificuldade para se impor e uma visão negativa de si mesma, geralmente, são perseguidos sem causa aparente ou revelada pelo autor do bullying, expostos às situações de constrangimento, humilhação e hostilidade (Manzini \& Branco, 2017; Souza, Pereira, \& Lourenço, 2011). Além disso, apresentam características físicas ou psicológicas que divergem dos grupos como: baixa 
estatura, obesidade, dificuldade de aprendizagem, uso de óculos entre outros (Salmivalli et al., 1996).

Quando o estudante sofre agressões de colegas e, em outro momento, pratica-o, é denominado alvo-autor (Lopes Neto, 2011). O alvo-autor reage ao bullying de forma agressiva, para a autoproteção com exposição a outras formas de violência que se levadas ao extremo, podem utilizar de armas branca ou de fogo para buscar justiça (Lisboa, Braga, \& Ebert, 2009; Salmivalli et al., 1996). Além disso, são os mais propensos ao consumo de drogas ilícitas e possuem indícios de sintomas depressivos, pensamentos suicidas e distúrbios psiquiátricos (Lopes Neto, 2011). A testemunha se apresenta em três tipos de comportamentos: 1) ser o assistente do autor e o reforçador que estimula a pratica das agressões; 2) aqueles que evitam ou ignorar o bullying; e 3) os defensores dos que sofrem os atos violentos (Hoffmann, 2012; Salmivalli et al., 1996).

As formas de identificar a prática de bullying podem levar em consideração dois fatores predisponentes, que Lopes Neto (2011) denomina de individuais e contextuais. Referentes aos individuais, estão associados o gênero (diferenças na prática entre masculino e feminino), a idade (alterações das ações em várias faixas etárias), comportamento exteriorizado (ações que fogem do controle), sintomas internalizados (alterações menos explícitas como introversão, depressão, ansiedade), competência social (relacionado às habilidades sociais), autopercepção (autoestima, autoimagem), percepções sobre os outros (empatia) e o desempenho acadêmico. Nos aspectos contextuais, o autor apresenta o ambiente familiar (conflito com os pais), escolar, comunitário (índices de violência, indicadores socioeconômicos), status social (isolamento, popularidade) e influência dos pares (valorização dos comportamentos apropriados e inapropriados).

Em relação aos contextos sociofamiliares dos envolvidos no bullying, Manzini e Branco (2017) descrevem que a família não é a única causadora da agressividade da criança ou do adolescente. Os autores citam que um estudante exposto por cenas agressivas pode favorecer o desenvolvimento de comportamentos hostis contra os pares e sentimentos de submissão, impondo o poder e a vontade dos mais fortes nas relações interpessoais. Por outro lado, levam em consideração, as múltiplas influências sobre o desenvolvimento humano e as possibilidades de caminhos a serem construídos, conforme os recursos que a sociedade, a família e a escola oferecem para o indivíduo. 
As consequências geradas pelas atitudes do bullying prejudicam a todos os indivíduos, quando não há esses recursos, por atingirem as estruturas cognitivas, sociais e afetivas, cujas ações fazem o estudante se avaliar de modo negativo e apresentar problemas de saúde, processo de ensino-aprendizagem e desenvolvimento psicossocial (Zequinão, Medeiros, Lise, Trevisol, \& Pereira, 2019). Diante disso, algumas pesquisas demonstram que estudantes envolvidos com o bullying têm maior tendência a desenvolver transtornos mentais, como ansiedade elevada, depressão, transtorno obsessivo-compulsivo, ideação suicida, suicídio, além de extrema sensibilidade, medo, vergonha, dificuldade de aprendizagem, baixo rendimento escolar, autoflagelação, comportamentos antissociais, uso de drogas e delinquência, porte de armas de fogo, redução da autoestima, evasão escolar, expressão das mesmas condutas violentas em sua vida adulta entre outros (Bandeira \& Hutz, 2012; Gloppen, McMorris, Gower, \& Eisenberg, 2018; Lopes Neto, 2011; Pham, Schapiro, John, \& Adesman, 2017; Piquero, Piquero, \& Underwood, 2017; Sampaio, Santos, Oliveira, Silva, \& Silva., 2015; Van \& Roseth, 2018).

Os estudos citados apontaram para prejuízos na saúde do estudante decorrente dos episódios de bullying. As agressões interferem, de forma negativa, o modo como o indivíduo se percebe e sente em relação a si e ao outro, afetando o comportamento (Bandeira \& Hutz, 2010). Sendo assim, são necessários aspectos avaliativos que promovam o enfrentamento desse tipo de violência e contribuam para a promoção do bem-estar e da qualidade de vida, como a autoestima (Schultheisz \& Aprile, 2013). A autoestima é um conjunto de sentimentos relacionado à aprovação ou à desaprovação, sendo um produto de autoavaliação (Silva, 2019). Ela é relevante para o desenvolvimento do indivíduo a partir da valorização de si e do outro, pois uma boa autoavaliação propõe maior probabilidade em assumir papéis ativos em grupos sociais e expressar suas percepções de maneira assertiva (Coopersmith, 1989; Rosenberg, 1965).

A autoestima envolve aspectos afetivos e cognitivos que resultam em um comportamento positivo ou negativo (Hutz \& Zanon, 2011). Uma valência positiva desta variável é fundamental para o indivíduo, por incluir sentimentos de valorização de si, englobando a satisfação e o orgulho pessoal, para que possa desenvolver novas aptidões de forma saudável para combater o bullying (Bandeira \& Hutz, 2010). Por isso, tais níveis geralmente indicam melhorias na saúde mental, nas habilidades sociais e promovem o bem-estar. Já uma avaliação negativa inclui sentimentos relacionados à desvalorização e 
uma visão negativa de suas qualidades pessoais, associados à depressão, à ansiedade, aos transtornos alimentares e à ideação suicida.

A autoestima é uma variável crítica e alguns autores citam os riscos de possuir uma autoestima elevada de forma exagerada. Uma elevada autoestima, quando desenvolvida de maneira equivocada, pode levar aos mais variados transtornos antissociais como o narcisismo, a arrogância, o orgulho e a soberba, passando a afetar as relações interpessoais de modo negativo (Bushman, Baumeister, Thomaes, Ryu, Begeer, \& West, 2009; Voli, 1998). A autoavaliação é diferente para cada indivíduo por estar inserida em outros fatores como o desempenho escolar, o tipo de interação entre professores e outros colegas, a estrutura física, a qualidade da escola e o ambiente em sala de aula (Lopez Júnior, 2010).

Essa construção da autoestima é constituída por meio de uma autoimagem que serve como base para a sua projeção (Muniz, 2018). O mesmo autor ressalta que as variáveis estarão vinculadas ao conhecimento individual de si mesmo e ao desenvolvimento das próprias potencialidades, percepções afetivas, atitudes e ideias de cunho pessoal, sendo importante diferenciá-las. A autoimagem está associada ao modo de perceber a si mesmo em caráter descritivo, na autoestima, o sujeito avalia todo o contexto. Além disso, ambos são construtos que se relacionam para a formação do autoconceito, produto das relações sociais para a constituição do self (Mendes, Dohms, Lettnin, Zacharias, \& Mosquera, 2012).

O self está contido no processo avaliativo da autoestima, e se relaciona as seguintes questões: 1) de identidade e de se sentir o mesmo, considerando o sujeito de constantes mudanças; 2) de se sentir único e o mesmo como qualquer outro; e 3 ) se a pessoa constrói o mundo do jeito que é ou se é construída como o mundo é (Bamberg \& Zielke, 2007). Estes pontos impactam nas definições de autoestima e autoconceito, já que, embora as variáveis estejam associadas, são distintas, pois o autoconceito é voltado para o autoconhecimento de si para os fatores externos, enquanto a autoestima corresponde ao modo como o indivíduo enfrenta o mundo e como percebe seus sentimentos de forma intrínseca. A identidade, as relações familiares e o self desempenham um papel fundamental na formação e na aceitação que os indivíduos têm de si mesmos e favorecem a autoestima positiva (Schultheisz \& Aprile, 2013).

Todos esses pontos têm relação com a personalidade do indivíduo, o que pode apontar a autoestima como um dos principais construtos por influenciar no modo como o 
indivíduo avalia seu meio (Del Vale, 2019). A variável se apresenta de duas formas: a implícita, que se refere a uma avaliação que ocorre de forma não intencional e fora da atenção consciente; e a explícita, relacionada ao autovalor consciente e deliberado, que o indivíduo faz sobre si mesmo. Ambos integram os fatores positivos e negativos que causam efeitos nos sentimentos, pensamentos e comportamentos do sujeito (Buhrmester, Swann, \& Blanton, 2011; Rudman, Dohn, \& Fairchild, 2007).

Quando os indivíduos são discriminados e desvalorizados pela sociedade, por algum atributo que possuem, são julgados pelo grupo como dispensáveis (Ronzani, Noto, \& Silveira, 2015). Um exemplo dessa característica está nos padrões de estética que reproduzem situações de preconceito do que é considerado belo, normal e desejável para um determinado grupo, o que pode acarretar diminuição da autoestima (Abramovay, 2015). Dessa forma, é possível que tais consequências permaneçam e prejudiquem a qualidade de vida do indivíduo (Schultheisz \& Aprile, 2013).

Tais variáveis podem afetar negativamente a autoestima dos estudantes, como o gênero, a cor da pele e o peso. Nas pesquisas de Bandeira e Hutz (2010) e Brito e Oliveira (2013), o gênero foi uma fonte motivacional para a ocorrência do bullying e causam prejuízos em aspectos psicológicos e emocionais nos alvos da agressão. Tanto os garotos como as garotas, em ambos os estudos, apresentaram baixos níveis de autoestima e interferências nos pensamentos, nas emoções e nos comportamentos dos estudantes, cujas avaliações de si diminuem, percebendo-se como fracos, incompetentes, sem valor e com dificuldade de romper o bullying (Fante, 2011; Chaves \& Souza, 2018).

A raça é outro aspecto que suscita as práticas de bullying. No estudo realizado por Gatto, Garbin, Corrente e Garbin (2017), foram constatados que a cor da pele não branca foi uma variável significativa, no qual estudantes com esta característica e que sofreram agressões, apresentaram duas vezes mais chances de ter uma autoestima negativa. O peso causa implicações psicológicas e interferem na autoestima, com indícios de baixos níveis, pois os estudantes obesos sofrem agressões verbais e se sentem excluídos dos grupos ou ridicularizados devido a insatisfação corporal ou capacidades físicas (Feldmann, Mattos, Halpern, Rech, Bonne \& Araújo, 2009). Além disso, indivíduos com menores índices de autoestima têm maiores chances de desencadear sintomas associados aos transtornos mentais, alertando o cuidado com a saúde mental (Paixão, Patias, \& Dell'Aglio, 2018).

O suporte social saudável é apresentado como mediador positivo para a autoestima, uma vez que minimiza as consequências geradas pelo bullying (Kleiman \& Riskind, 
2013). Na pesquisa de Souza, Araújo e Pereira (2016), buscou-se entender a relação entre os comportamentos de bullying com a autoestima e a qualidade do suporte social. Os resultados indicam que, quanto maiores as práticas de violência, menores serão os níveis de autoestima e suporte social, além de apresentar sofrimento psicológico tanto para os que praticam como para quem sofre.

Outra forma de contribuição para uma autoestima positiva são as habilidades sociais, pois potencializam as funções cognitivas, sociais e psicológicas, por meio de técnicas que promovem o enfrentamento das situações, sendo importantes no processo de desenvolvimento do indivíduo (Del Prette \& Del Prete, 2011). Além disso, as habilidades sociais são importantes nas intervenções contra o bullying, pois podem lidar com as agressões de forma saudável, melhorando o relacionamento interpessoal e a qualidade de vida (Silva, Oliveira, Carlos, Lizzi, Rosário, \& Silva, 2018). Um baixo índice de habilidades sociais causa prejuízos nos comportamentos relacionadas à empatia, à desenvoltura social, assertividade, autocontrole e civilidade, tanto os autores como os alvos do bullying, demonstrando a importância de compreender e intervir em todos os envolvidos (Terroso, Wendt, Oliveira, \& Argimon, 2016). Diante desta consideração, torna-se importante entender o impacto do bullying e a ampliação de pesquisas relacionadas com a autoestima, por estar associado com o bem-estar do indivíduo e as percepções e autoavaliações daqueles que praticam e sofrem as agressões.

\section{Considerações finais}

O presente artigo teve o objetivo de apresentar e discutir os principais conceitos, as relações e distinções sobre bullying, preconceito e autoestima. A hipótese levantada foi atingida de forma positiva, pois notou-se que o preconceito e o bullying são duas formas de violência escolar especificas que têm conceitos voltados para a relação de poder, articulados com a discriminação e o domínio sobre o outro, e que provocam problemas emocionais e físicos, prejudicando a autoestima. Além disso, verificou-se uma preocupação no que se refere às essas condutas agressivas, nas quais os estudantes percebem os fenômenos como natural ou uma forma de "brincadeiras" entre os grupos, o que pode ocasionar o seu aumento em curto e longo prazo.

Os modelos teóricos ressaltaram que os alunos justificam suas ações agressivas com motivos que acreditam não relacionar com o preconceito, o que pode ser um ponto de 
partida para o crescimento da discriminação dentro das escolas. Os estudos empíricos reforçam essa conclusão e apresentam as consequências desses atos que podem gerar desgaste emocional nos alvos, provocando baixa autoestima, ansiedade, depressão, evasão escolar, suicídio entre outros. Além disso, notou-se que a autoestima é uma variável relacionada com o processo de saúde-doença, sendo necessário identificar os fatores que diminuem o autovalor do estudante em aspectos cognitivos, sentimentais e comportamentais, para preservar a saúde mental, a partir da valorização da afetividade e das condutas que capacitem e construam uma relação interpessoal mais saudável.

Cabe ressaltar que há poucos estudos brasileiros sobre a relação entre bullying e autoestima dos envolvidos com o bullying, sendo necessário uma investigação mais minuciosa dessas temáticas. Sobre as limitações deste artigo, não houve uma investigação profunda sobre o gênero, a idade ou raça, sendo, estes, variáveis que influenciam diretamente a autoestima dos estudantes.

Com isso, os resultados da pesquisa podem ser relevantes, por oferecer uma reflexão sobre o preconceito e o bullying nas escolas, o papel da autoestima dentro desse campo e como os fatores socioculturais afetam as condutas daqueles que praticam e sofrem com a agressão. Espera-se que o estudo apresente as possibilidades de análise dos fenômenos e que intervenções sejam realizadas para aumentar a autoestima, pois, notouse que uma avaliação positiva, de si e do outro, contribui para o enfrentamento das condutas agressivas, através de habilidades sociais que favoreçam a qualidade de vida e o bem-estar psíquico e emocional.

\section{Referências}

Abramovay, M. (2015). Programa de prevenção a violência nas escolas. Brasília: Apresentação da Faculdade Latino Americano de Ciências Sociais (FLACSO). Recuperado de http://flacso.org.br/?s=violência+nas+escolas

Antunes, D. C. (2010). Bullying, Razão Instrumental e Preconceito. São Paulo: Casa do Psicólogo.

Antunes D. C., \& Zuin, A. A. S. (2008). Do Bullying ao Preconceito: Os desafios da barbárie à educação. Psicologia e Sociedade, 20(1), 33-41. Recuperado de https://www.academia.edu/4330641/Do_bullying_ao_preconceito_os_desafios_da_b arbárie à educação

Bamberg, M., \& Zielke, B. (2007). From dialogical practices to polyphonic thought? Developmental inquiry and where to look for it. International Journal for Dialogical 
Sciences, 2(1), 223-242. Recuperado de https://www.researchgate.net/publication/252174230 FROM DIALOGICAL_PRA CTICES TO POLYPHONIC THOUGHT DEVELOPMENTAL INQUIRY AND WHERE TO LOOK FOR IT

Bandeira, C. M., \& Hutz, C. S. (2010). As implicações do Bullying na autoestima dos adolescentes. Revista Semestral da Associação Brasileira de Psicologia Escolar e Educacional, 14(1), 131-138. Recuperado de https://www.scielo.br/pdf/pee/v14n1/v14n1a14

Bandeira, C. M., \& Hutz, C. S. (2012). Bullying: prevalência, implicações e diferenças entre os gêneros. Psicologia Escolar e Educacional, 16(1), 35-44. Recuperado de https://www.scielo.br/scielo.php?pid=S1413$\underline{85572012000100004 \& \text { script }=\text { sci arttext }}$

Binsfeld, A. R., \& Lisboa, C. S. M. (2010). Bullying: Um estudo sobre papéis sociais, ansiedade e depressão no contexto escolar do Sul do Brasil. Interpersona, 4(1), 74105. https://www.researchgate.net/publication/49595042_Bullying_Um_estudo_sobre_pa peis_sociais_ansiedade_e depressao_no_contexto_escolar

Bodenhausen, G.V., \& Richeson, J. A. (2010). Prejudice, Stereotyping, and Discrimination. In R. F. Baumeister \& E. J. Finkel (Eds.), Advanced Social Psychology: The State of the Science (pp. 341-383). New York: Oxford University Press. Recuperado de https://www.scholars.northwestern.edu/en/publications/prejudice-stereotyping-anddiscrimination

Brito, C. C., \& Oliveira, M. T. (2013). Bullying and self-esteem in adolescents from public schools. Jornal de Pediatra, 89(6), 601-607. Recuperado de https://www.sciencedirect.com/science/article/pii/S225555361300116X

Buhrmester, M., Swann, W. B., \& Blaton, H. (2011). Implicit self-esteem: Nature, measurement, and a new way forward. Journal of Personality and Social Psychology, 100(2), 365-385. Recuperado de https://www.researchgate.net/publication/47642315_Implicit_SelfEsteem_Nature Measurement and_a_New Way_Forward

Bushman, B. J., Baumeister, R. F., Thomaes, S., Ryu, E., Begeer, S., \& West, S. (2009). Looking again, and harder, for a link between low self-esteem and aggression. Journal of Personality, 77(2), 424-446. Recuperado de https://onlinelibrary.wiley.com/doi/abs/10.1111/j.1467-6494.2008.00553.x

Chaves, D. R. L., \& Souza, M. R. (2018). Bullying e Preconceito: A atualidade da barbárie. Revista Brasileira de Educação, 23, e230019, 1-17. Recuperado de https://www.scielo.br/scielo.php?pid=S141324782018000100214\&script $=$ sci abstract\&tlng $=$ pt

Coopersmith, S. (1989). Coopersmith - self-esteem Inventory. Palo Alto, CA: Consulting Psychologists Press. 
Cordeiro, A. F., M., \& Buendgens, J. F. (2012). Preconceitos na escola: Sentidos e Significados atribuídos pelos adolescentes no Ensino Médio. Psicologia Escolar e Educacional, $\quad$ 16(1), p.45-54. Recuperado de https://www.scielo.br/scielo.php?pid=S1413$\underline{85572012000100005 \& \text { script }=\text { sci abstract\&tlng }=\text { pt }}$

Crandall, C. S., \& Eshleman, A. (2003). A justification suppression model of the expression and experience of prejudice. Psychological Bulletin, 129(3), 414-446. Recuperado de https://psycnet.apa.org/record/2003-00782-007

Crochík, J. L. (2015). Formas de violência escolar: Preconceito e Bullying. Movimento, 3(2), 29-56. Recuperado de http://www.periodicos.uff.br/revistamovimento/article/view/32559/18694

Crochík, J. L. (2016). Educação inclusiva: algumas pesquisas. Saarbrücken: Novas Edições Acadêmicas.

Crochík, J. L. (2019). Preconceito e Bullying: marcas de regressão psíquica socialmente induzida. Psicologia USP, 30, e190006. Recuperado de http://www.scielo.br/scielo.php?script=sci arttext\&pid=S010365642019000100215\&lng=en\&nrm=iso

Crochík, J. L., \& Crochík, N. (2017). Bullying, Preconceito e Desempenho Escolar: uma nova perspectiva. São Paulo: Benjamim Editorial.

Del Prette, A., \& Del Prette, Z. A. P. (2011). Habilidades sociais: intervenções efetivas em grupo. São Paulo, SP: Casa do Psicólogo.

Del Vale, G. F. (2019). Influência de parâmetros biométricos e sociodemográficos na resiliência, autoestima, satisfação e percepção corporal de adolescentes do ensino fundamental II. Dissertação de Mestrado, Programa de Pós-Graduação em Enfermagem, Universidade Federal de São Carlos, São Paulo. Recuperado de https://repositorio.ufscar.br/handle/ufscar/11231

Dovidio, J. F., Hewstone, M., Glick, P., \& Esses, V. M. (2010). Prejudice, Stereotyping and Discrimination: Theoretical and Empirical Overview. In: J. F. Dovidio, M. Hewstone, P. Glick, \& V. M. Esses (Eds). The SAGE handbook of prejudice, stereotyping and discrimination (pp. 3-28). London: SAGE. Recuperado de https:/www.semanticscholar.org/paper/Prejudice\%2C-stereotyping-anddiscrimination $\% 3 \mathrm{~A}$-and-Dovidio Hewstone/b1cea4d91d9593ba5b9790c8159bf35004cd8ac5

Fante, C. (2005). Fenômeno Bullying: como prevenir a violência nas escolas e educar para a paz. São Paulo: Verus.

Fante, C. (2011). Fenômeno Bullying (6a ed.), Campinas-SP: Verus.

Feldmann, L. R. A., Mattos, A. P., Halpern, R., Rech, R., Bonne, C. C., \& Araújo, M. B. (2009). Implicações psicossociais na obesidade infantil em escolares de 7 a 12 anos de 
uma cidade Serrana do Sul do Brasil. Revista Brasileira de Obesidade, Nutrição e Emagrecimento, 3(15), 225-233. Recuperado de https://dialnet.unirioja.es/servlet/articulo? codigo $=4837828$

Gatto, R. C. J., Garbin, A. J. I., Corrente, J. E., \& Garbin, C. A. S. (2017). Self-esteem level of Brazilian teenagers victims of bullying and its relation with the need of orthodontic treatment. Revista Gaúcha Odontologia, 65(1), 30-36. Recuperado de https://www.scielo.br/scielo.php?script=sci arttext\&pid=S1981$\underline{86372017000100030}$

Gloppen, K., McMorris, B., Gower, A., \& Eisenberg, M. (2018). Associations between bullying involvement, protective factors, and mental health among American Indian youth. American Journal of Orthopsychiatry, 88(4), 413-421. Recuperado de https://www.ncbi.nlm.nih.gov/pubmed/28816487

Herrera Rubalcaba, D., \& Sánchez Quintero, C. (2013). Tipología y grado de prejuicio de un grupo de españoles hacia el colectivo colombiano. Una lectura con perspectiva de género. Educación Y Humanismo, 15(24), 236-247. Recuperado de http://revistas.unisimon.edu.co/index.php/educacion/article/view/2218

Hoffmann, C. (2012). Bystanders in Bullying situations: Differences between participant troles and their reactioins to manipulation. Dissertação de Mestrado, Department Psychology of Conflict Risk and Safety, University of Twente, Países Baixos. Recuperado de https://www.semanticscholar.org/paper/Bystanders-in-bullyingsituations \%3A-differences-and-

Hoffmann/ec6566c13e6902f1e8380739e549b9e24831528c

Horkheimer, M., \& Adorno, T. W. (1985). Dialética do esclarecimento. $2^{\circ}$ ed. Rio de Janeiro: Jorge Zahar.

Hutz, C. S., \& Zanon, C. (2011). Revisão da adaptação, validação e normatização da escala de autoestima de Rosenberg. Avaliação Psicológica, 10(1), 41-49. Recuperado de $\quad$ http://pepsic.bvsalud.org/scielo.php?script=sci arttext\&pid=S1677$\underline{04712011000100005}$

Imhoff, D., Dreizik, M., \& Brussino, S. (2020). Análisis psicosocial del prejuicio hacia trabajadoras sexuales. $C S, \quad$ (30), 173-196. Recuperado de http://www.scielo.org.co/scielo.php?pid=S2011$\underline{03242020000100173 \& \text { script }=\text { sci abstract\&tlng }=\text { en }}$

Instituto Brasileiro de Geografia e Estatística (2015). Pesquisa Nacional de Saúde do Escolar (PeNSE). Rio de Janeiro: IBGE. Relatório de pesquisa. Recuperado de https://www.ibge.gov.br/estatisticas/sociais/populacao/9134-pesquisa-nacional-desaude-do-escolar.html

Instituto Nacional de Estudos e Pesquisas Educacionais Anísio Teixeira. (2019). Pesquisa Internacional sobre Ensino e Aprendizagem revela impacto do bullying nas escolas. (INEP). Recuperado de http://portal.inep.gov.br/artigo//asset publisher/B4AQV9zFY7Bv/content/pesquisa-internacional-sobre-ensino-eaprendizagem-revela-impacto-do-bullying-nas-escolas/21206 
Kleiman, E. M., \& Riskind, J. H. (2013). Utilized social support and self-esteem mediate the relationship between perceived social support and suicide ideation. A Test of a Multiple Mediator Model. Crisis, 34(1), 42-49. Recuperado de https://www.ncbi.nlm.nih.gov/pubmed/22846448

Krech, D., Crutchfield, R. S., \& Ballachey, E. L. (1975). O individuo na sociedade: um manual de psicologia social. São Paulo: Pioneira.

Lei $n^{\circ} 13.185$, de 6 de novembro de 2015. (2015, 6 de novembro). Institui o Programa de Combate à Intimidação Sistemática (Bullying). Diário Oficial da União: seção 1. Recuperado de http://www.planalto.gov.br/ccivil_03/_ato20152018/2015/lei/113185.htm

Lima, M. E. O. (2011). Preconceito. In L. Camino, A. R. R. Torres, M. E. O. Lima, \& M. E. Pereira (Eds). Psicologia Social: Temas e Teorias (pp. 587-640). Brasília: Technopolitik.

Lisboa, C. S. (2005). Comportamento agressivo, vitimização e relações de amizade de crianças em idade escolar: fatores de risco e proteção. Tese de Doutorado, Universidade Federal do Rio Grande do Sul, Porto Alegre. Recuperado de https://www.lume.ufrgs.br/handle/10183/6875

Lisboa, C. S., Braga, L. L., \& Ebert, G. (2009). O fenômeno bullying ou vitimização entre pares na atualidade: definições, formas de manifestação e possibilidades de intervenção. Contextos Clínicos, 2(1), 59-71. Recuperado de http://pepsic.bvsalud.org/scielo.php?script=sci arttext\&pid=S1983$\underline{34822009000100007 \& \operatorname{lng}=p t \& t \operatorname{lng}=p t .}$

Lopes Neto, A. A. (2011). Bullying: Saber Identificar e Como Prevenir. São Paulo: Brasiliense.

Lopes Neto, A. A., \& Saavedra, L. H. (2004). Diga não ao bullying: Programa de Redução do Comportamento Agressivo entre Estudantes. Rio de Janeiro: Abrapia.

Lopez Junior, P. F. (2010). A Autoestima e sua influência no espaço escolar, com a atuação dos orientadores educacional e pedagógico. Monografia, Pós-Graduação "Lato sensu", Curso de Orientação Educacional e Pedagógica, Universidade Candido Mendes, Rio de Janeiro. Recuperado de http://www.avm.edu.br/monoteca.asp?tipo

Malta, D. C., Mello, F. C. M., Prado, R. R., Sá, A. C. M. G. N., Marinho, F., Pinto, I. V., Silva, M. M. A., \& Silva, M. A. I. (2019). Prevalência de bullying e fatores associados em escolares brasileiros, 2015. Ciência \& Saúde Coletiva, 24 (4), 1359-1368. Recuperado de http://www.scielo.br/scielo.php?script=sci arttext\&pid=S141381232019000401359

Manzini, R. G. P., \& Branco, A. U. (2017). Bullying: Escola e Família Enfrentando a Questão. Porto Alegre, RS: Mediação. 
Martins, J. J., \& Kämpf, R. (2014). Preconceito e Repetição: Diferentes maneiras de entender o Bullying. Curitiba, PR: Positivo.

Mendes, A. R., Dohmsn, K. P., Lettnin, C., Zacharias, J., Mosquera, J. J. M., \& Stobäus, C. D. (2012). Autoimagem, Autoestima e Autoconceito: Contribuições pessoais e profissionais na docência. Seminário de Pesquisa em Educação da Região Sul. São Paulo. Recuperado de https://m.scirp.org/index.html

Muniz, J. (2018). Desempenho Acadêmico: Um estudo sobre a influência da Autoestima no rendimento escolar de discentes dos últimos anos do Ensino Fundamental II. Dissertação de Mestrado, Programa Stricto Sensu em Educação, Universidade Católica de Brasília, Brasília. Recuperado de http://bdtd.ibict.br/vufind/Record/UCB c1927a0bb4448af6ee9b65962c9eefcd

Nunes, S. S. (2010). Racismo contra negros: um estudo sobre o preconceito sutil. Tese de Doutorado, Instituto de Psicologia da Universidade de São Paulo, São Paulo. Recuperado de https://www.teses.usp.br/teses/disponiveis/47/47131/tde-27072010082636/pt-br.php

Oliboni, S. P., Lunardi, V. L., Lunardi, G. L., Pereira, B. O., \& Oliveira, W. A. (2019). Prevalência do bullying entre alunos do ensino fundamental. Aletheia, 52 (1), 8-21. Recuperado de http://www.periodicos.ulbra.br/index.php/aletheia/article/view/5278

Olweus, D. (1993). Bullying at School. What we know and what we can do. Oxford, UK: Blackwell.

Olweus, D. (2013). School Bullying: Development and some important challenges. Annual Review of Clinical Psychology, 9(1), 751-80. Recuperado de: https://www.ncbi.nlm.nih.gov/pubmed/23297789

Paixão, R. F., Patias, N. D., \& Dell'Aglio, D. D. (2018). Autoestima e Sintomas de Transtornos Mentais na Adolescência: Variáveis Associadas. Psicologia: Teoria e Pesquisa, 34(1), e34436, p. 1-8. Recuperado de https://www.scielo.br/scielo.php?script=sci_arttext\&pid=S0102$\underline{37722018000100535 \& \operatorname{lng}=\text { en\&nrm }=\text { iso\&tlng }=\mathrm{pt}}$

Parker, R. (2012), Stigma, Prejudice and Discrimination in global public health. Caderno de Saúde Pública, 28(1), 164-169. Recuperado de http://www.scielo.br/scielo.php?script=sci arttext\&pid=S0102-311X2012000100017

Pereira, C. R., Vala, J. A., \& Costa-Lopes, R. (2010). From prejudice to discrimination: the legitimizing role of perceived threat in discrimination against immigrants. European Journal of Social Psychology, 40(7), 1231-1250. Recuperado de https://onlinelibrary.wiley.com/doi/abs/10.1002/ejsp.718

Pettigrew, T. F., \& Meertens, R. W. (1995). Subtle and blatant prejudice in Western Europe. European Journal of Social Psychology, 25(1), 57-75. Recuperado de https://psycnet.apa.org/record/1995-28884-001 
Pham, T. B., Schapiro, L. E., John, M., \& Adesman, A. (2017). Weapon carrying among victims of bullying. Pediatrics, 140(6), e20170353. Recuperado de https://www.ncbi.nlm.nih.gov/pubmed/29180461

Piquero, A. R., Piquero, N. L., \& Underwood, M. K. (2017). Correlates of and consequences for bully-victimis in a sample of serious adolescent offenders. Youth Violence and Juvenile Justice, 15(4), 359-373. Recuperado de https://journals.sagepub.com/doi/abs/10.1177/1541204016657396

Rigby, K. (1999). What harm does bullying do? Children and Crime: Victims and Offenders. 1-13. Recuperado de https://www.researchgate.net/publication/242084964_WHAT_HARM_DOES_BUL LYING DO

Rocha, W. A. R., Paixão, L. V. J., Teixeira, J. A. L., Paixão, N. E., Soares, C. F., \& Rocha, K. L. F. (2019). Bullying na escola: Enfrentamento na perspectiva do docente. Revista Psicologia e saberes, 8(11), 279-304. Recuperado de https://revistas.cesmac.edu.br/index.php/psicologia/article/view/986

Rodkin, P. C. R., Espelage, D. L., \& Hanish, L. D. (2015). A relational framework for understanding bullying. Developmental antecedents and outcomes. American Psychologist, $\quad 70(4), \quad 311-321 . \quad$ Recuperado de https://pubmed.ncbi.nlm.nih.gov/25961312/

Rosenberg, M. (1965). Society and the adolescent self-image. Princeton: Princeton University Press.

Rother, E. T. (2007). Revisão sistemática x revisão narrativa. Acta Paulista de Enfermagem, 20(2), 5-6. Recuperado de http://www.scielo.br/scielo.php?script=sci_arttext\&pid=S0103-21002007000200001

Rozani, T. M., Noto, A. R., \& Silveira, P. S. (2015). Reduzindo o Estigma entre Usuários de Drogas: Guia para profissionais e gestores. Editora UFJF: Juiz de Fora.

Rudman, L. A., Dohn, M. C., \& Fairchild, K. (2007). Implicit self-esteem compensation: automatic threat defense. Journal of personality and social psychology, 93(5), 798813. Recuperado de https://pubmed.ncbi.nlm.nih.gov/17983301/

Salmivalli, C., Lagerspetz, K., Björkqvist, Österman, K., \& Kaukiainen, A. (1996). Bullying as a group process: Participant roles and their relations to social status within the group. Aggressive Behavior, 22(1), 1-15. Recuperado de https://onlinelibrary.wiley.com/doi/abs/10.1002/\%28SICI\%291098$2337 \% 281996 \% 2922 \% 3 \mathrm{~A} 1<1 \% 3 \mathrm{~A} \% 3 \mathrm{AAID}-\mathrm{AB} 1>3.0 . \mathrm{CO} \% 3 \mathrm{~B} 2-\mathrm{T}$

Sampaio, J. M. C., Santos, G. V., Oliveira, W. A., Silva, J. L., \& Silva, M. A. I. (2015). Prevalência de bullying e emoções de estudantes envolvidos. Texto Contexto Enfermagem, 24(2), 344-352. Recuperado de https://www.scielo.br/scielo.php?pid=S0104$\underline{07072015000200344 \& \text { script }=\text { sci abstract\&tlng }=\text { pt }}$ 
Schultheisz, T. S. V., \& Aprile, M. R. (2013). Autoestima, conceitos correlatos e avaliação. Revista Equilíbrio Corporal e Saúde, 5(1), 36-48. Recuperado de https://www.academia.edu/12178758/AUTOESTIMA_CONCEITOS_CORRELAT OS E AVALIACÕES

Smith, P., \& Sharp, S. (1994). School bullying: insights and perspectives. New York: Routledge.

Silva, D. A. (2019). A autoestima e o comportamento suicida em estudantes universitários: uma revisão de literatura. Revista Eletrônica Acervo Saúde, 23(1), 1-6. Recuperado

de https://www.acervomais.com.br/index.php/saude/article/view/422/435

Silva, J. L., Oliveira, W. A., Carlos, D. M., Lizzi, E. A. S., Rosário, R., \& Silva, M. A. I. (2018). Intervenção em habilidades sociais e bullying. Revista Brasileira de Enfermagem, 71(3), 1085-1091. Recuperado de https://www.scielo.br/scielo.php?script=sci arttext\&pid=S0034$\underline{71672018000301085 \& \operatorname{lng}=\mathrm{en} \& \mathrm{nrm}=\mathrm{iso} \& \mathrm{t} \operatorname{lng}=\mathrm{pt}}$

Sousa, M. D., Araújo, B., \& Pereira, B. (2016). Bullying, Autoestima e Suporte social, que relação? In B. Pereira, A. J. G. Barbosa, \& L. M. Lourenço (Eds). Estudos sobre Bullying: Família, Escola e Atores (pp.163-177). Recuperado de https://repositorium.sdum.uminho.pt/handle/1822/43350

Souza, J. M. (2013). Bullying: uma das faces do preconceito no contexto escolar. Dissertação de Mestrado, Programa de Pós-Graduação em Psicologia Social, Universidade Federal de Sergipe, Sergipe. Recuperado de https://www.repositorio.ufs.br/handle/riufs/6013

Souza, J. M., Silva, J. P., \& Faro, A. (2015). Bullying e Homofobia: Aproximações Teóricas e Empíricas. Psicologia Escolar e Educacional, 19(2), 289-298. Recuperado de $\quad$ http://www.scielo.br/scielo.php?script=sci arttext\&pid=S1413$\underline{85572015000200289}$

Souza, R., Pereira, B. O., \& Lourenço, L. M. (2011). O bullying, locais e representações dos recreios: Estudos com crianças de uma escola básica de $5^{\circ}$ e $6^{\circ}$ anos. In A. J. Barbosa, L. M. Lourenço, \& M. B. Pereira (Eds.). Bullying Conhecer e Intervir (pp. 33-49). Minas Gerais: UFJF.

Terroso, L. B., Wendt, G. W., Oliveira, M. S., \& Argimon, I. I. L. (2016). Habilidades sociais e Bullying em adolescentes. Temas em psicologia, 24(1), 251-259. Recuperado de $\quad$ http://pepsic.bvsalud.org/scielo.php?script $=$ sci arttext\&pid=S1413$\underline{389 X 2016000100013}$

Van Ryzin, M. J., \& Roseth, C. J. (2018). Cooperative learning in middle school: A means to improve peer relations and reduce victimization, bullying, and related outcomes. Journal of Educational Psychology, 110(8), 1192-1201. Recuperado de https://psycnet.apa.org/record/2018-08642-001 
Voli, F. (1998). La Autoestima del profesor, manual de reflexión y acción educativa. Madrid: PPC Editorial.

Zequinão, M. A., Medeiros, P., Lise, F. A., Trevisol, M. T. C., \& Pereira, M. B. F. L. O. (2019). Associação entre bullying escolar e o país de origem: Um estudo transcultural. Revista Brasileira de Educação, 24, e240013, 1-22. Recuperado de https://www.scielo.br/scielo.php?script=sci_arttext\&pid=S1413$\underline{24782019000100207}$

Zunino, L. M. R., Bastos, J. L. D., Coelho, I. Z., Mendes, F., \& Massignam, F. M. (2015). A discriminação no ambiente universitário: Quem, onde e por quê? Revista Saúde e Transformação Social, 6(1), 13-30. Recuperado de http://incubadora.periodicos.ufsc.br/index.php/saudeetransformacao/article/view/359 $\underline{5}$

Recebido em: 07/04/2020

Aprovado em: 01/06/2020 